\title{
Análisis de la teoría del Valor-Trabajo en el desempeño productivo de algunos sectores económicos venezolanos durante el año 2009: estudio de las transferencias de valor'
}

Análise da teoria do Valor no desempenho produtivo de alguns setores econômicos venezuelanos durante o ano de 2009: estudo das transferências de valor

\section{Luciana Madrid Cobeña Escuela Venezolana de Planificación}

\begin{abstract}
Resumen: En el presente artículo se plantea una demostración teórica y empírica sobre el proceso de las transferencias de valor entre algunos sectores de la economía venezolana para el año 2009, teniendo como eje de análisis a la Teoría del Valor-Trabajo. La metodología utilizada contempla analizar los sectores o ramas económicas que pueden ser considerados como productivos e improductivos, determinar el valor agregado generado y apropiado de algunos sectores, determinar y explicar el impacto del sector improductivo en los sectores seleccionados, para finalmente estimar las transferencias de valor en términos de horas trabajo. El análisis se basa en la identificación de una unidad de contabilización homogénea para determinar el excedente que se transfiere de un sector o rama a otro, según las actividades que cada uno realiza. Tomando en cuenta la particularidad rentista de la economía venezolana, se utilizan aproximaciones gruesas a efectos de realizar los cálculos correspondientes. Se espera con la investigación contribuir en el proceso de planificación en materia económica en el país.
\end{abstract}

Palabras claves: Teoría del valor trabajo; Transferencias de valor; Trabajo productivo.

Resumo: O presente artigo realiza uma demonstração teórica e empírica sobre o processo de transferências de valor entre alguns setores da economia venezuelana para o período compreendido entre janeiro e dezembro do ano de 2009, para esta pesquisa foi utilizada como eixo de análise a Teoria do Valor-Trabalho. A metodologia utilizada inclui: a análise de setores ou ramos que podem ser considerados como produtivos e improdutivos no conjunto da economia venezuelana; determinar o valor agregado gerado e apropriado em alguns setores; determinar e explicar o impacto do setor improdutivo no conjunto dos setores selecionados, para finalmente estimar as transferências de valor em termos de horas de trabalho. A análise baseou na identificação de uma unidade homogênea de contabilidade para determinar o excedente que é transferido de um setor ou ramo para outro, conforme as atividades que cada setor executa. Considerando a particularidade rentista da economia venezuelana, foram utilizadas aproximações grossas para poder realizar os cálculos correspondentes. Espera-se com esta pesquisa contribuir com o processo de planejamento dentro da esfera econômica do país.

Palavras-chave: Teoria do valor trabalho; Transferências de valor; Produtivo-trabalho.

JEL: J24

\section{Introducción}

Los planteamientos de Marx en la Economía Política aparecen como una perspectiva que partiendo de la Clásica, va mucho más allá. Es dinámica y profunda, capta los rasgos esenciales del sistema (es difícil precisar en este análisis los rasgos presentes en el siglo XVII, sin embrago se hace referencia de manera general a las condiciones propias del modelo capitalista) y por esto, resulta más crítica. En Marx, la

1 Trabajo de Investigación realizado en la Escuela Venezolana de Planificación, como parte de la línea de investigación en Economía y como contribución para el fortalecimiento del desarrollo académico (investigación docencia y extensión) de la Institución. 
distinción de los rasgos más internos y esenciales, así como los más externos y menos decisivos de los procesos sociales, se evidencia con mayor intensidad que en los Clásicos.

Bajo este enfoque, aparecen nociones claves que determinan el centro de la Economía Política, a saber: la cuestión del trabajo productivo e improductivo ${ }^{2}$, la noción del valor, en tanto se habla de la representación del trabajo, pero no de cualquier trabajo, se hace referencia entonces a aquel que se da "solo en conjunción con un modo específico de organización social de la producción"3; y que se ve expresado en el tiempo (horas) que materialmente el hombre dedica a una actividad "productiva", entendiendo que el valor se mide en tiempo de trabajo, es decir en horas de trabajo empleadas.

$\mathrm{Al}$ respecto, Marx establece los determinantes del valor y se alude a su magnitud, a su tiempo de duración y a su unidad de medida en las distintas fracciones de tiempo (horas, días)4, dicho de otro modo esto será el tiempo de trabajo medio socialmente reconocido, que en lo sucesivo servirá de base y de sustancia para comprender la diferencia que se da al llevar a cabo el intercambio de productos en el mercado 5 y más expresamente para el análisis de las transferencias de valor. En este sentido, es a través de la Teoría del Valor-Trabajo que Marx expresa la representación del trabajo del hombre por el valor.

Para el análisis puntual sobre las transferencias de valor entre sectores, se parte de que surgen de las desviaciones de los precios de producción con respecto a los precios directos, esto es, de los precios correspondientes al valor social, que a su vez implica transferencias de valor en el interior de un sector determinado, ya que el valor social es en sí mismo el promedio de los valores individuales de diferentes ramas o sub-ramas. Es decir, las mercancías producidas bajo mejores condiciones que las del promedio tendrán valores individuales inferiores al valor social (medio), puesto que producirlas toma menor tiempo de trabajo que el promedio; por su parte aquellas producidas en peores condiciones que el promedio tendrán valores individuales más altos que el valor social.

Es por esto que al momento de plantear el intercambio entre las mercancías, surge entonces el problema de las transferencias, ya que los productores socialmente más ineficientes, en relación a la media, transferirán parte de sus recursos a otros sectores de la economía. De esta manera el punto de partida fundamental es la creación de valor y, al mismo tiempo, el papel del trabajo calificado que se ha empleado en la generación de mercancías.

Como ya se dijo, esta transferencia se realiza a través de los precios, sin embargo en una economía de mercado siempre funcionan dos unidades de contabilización: la de los precios, que es la visible, y la de los valores, que es más bien subyacente. Esto es, por un lado la unidad monetaria y, por el otro, la hora de trabajo social.

En el caso particular de Venezuela, una economía mono productora, extractivista, cuya base fundamental es la explotación de petróleo, se considera como un sistema de precios directos con presencia de sectores improductivos. A partir de este supuesto, se considera la definición de trabajo productivo e improductivo que hiciera Marx para

2“Es la base de toda la Economía Política” (Malthus, apud Marx, 1969-1971, parte I: 157). Tomado de Baptista en Itinerario por la Economía Política, 2008: 96.

3 “El trabajo en sí mismo no da valor al producto, sino sólo el trabajo que es organizado en determinada forma social” (Rubín, 1974:121).

4 Marx, 1973. Tercera Edición, tercera Reimpresión, 2006.

5Dos Santos, 1978.

Informe Gepec, Toledo, v. 18, n. 2, p. 137-146, jul./dez. 2014 
tratar de demostrar el efecto de la naturaleza de las actividades que se realiza en cada sector a la hora de transferir valor (monetario y de trabajo social) de un sector a otro.

La presencia del sector improductivo para Venezuela presenta algunas dificultades que hacen más complejo las precisiones a la hora de realizar los cálculos, sin embargo; es importante no perder de vista que las actividades improductivas, por definición, no producen valor, entonces el problema que emerge es el de cuánto valor se genera en cada sector, quienes lo absorben, cuales son los sectores considerados productivos e improductivos y el impacto de estos últimos sobre el resto de la economía.

En este sentido, a continuación se presenta de forma más o menos detallada la metodología utilizada para el análisis, así como los resultados y algunas consideraciones a manera de conclusiones.

\section{Algunos elementos metodológicos}

El punto básico es identificar las transferencias que van desde un sector o rama a otro. Para lo cual, se debe identificar una unidad de contabilización homogénea. Para el caso, los valores de todas las variables son específicos para el período 2009, lo usual es escoger un período calendario (un año, un quinquenio, un semestre, etc.). Se toma este año de referencia para observar el comportamiento de las transferencias de valor durante un período que muestra un descenso en el comportamiento del PIB después de veintidós trimestres consecutivos de crecimiento, y luego de la crisis que se desplegó con intensidad en la segunda mitad del año 2008 que afectó negativamente el desempeño económico de la gran mayoría de los países a escala mundial.

Según estimaciones realizadas por el Banco Central de Venezuela, para el período 2009 el producto interno bruto (PIB) experimentó, en términos reales, una contracción interanual de 2,9\%. Este resultado constituyó la primera caída que exhibió el PIB luego de 5 años de crecimiento. El menor nivel de actividad económica se observó particularmente en el sector petrolero, cuyo valor agregado se redujo a una tasa anual de $6,1 \%$, como consecuencia de los recortes de producción implementados por la OPEP, en un contexto de debilidad de la demanda energética producto de la crisis económica global.

El sector no petrolero registró un decrecimiento de 1,9\%, asociado, fundamentalmente, a las contracciones experimentadas por el valor agregado bruto (VAB) de las actividades manufactura $(7,2 \%)$, comercio y los servicios de reparación $(8,2 \%)$ y transporte y almacenamiento $(8,5 \%)$. Entre los factores que incidieron en estos resultados destaca el debilitamiento de la demanda agregada, la incertidumbre en cuanto a la recuperación de la economía mundial y la contracción que registraron las importaciones durante el año, en particular en el tercer y cuarto trimestre. En contraste con estos resultados, debe destacarse el acelerado ritmo de crecimiento que mantuvo el sector comunicaciones $(10,1 \%)$, así como también los avances registrados por la construcción $(3,1 \%)$, electricidad y agua $(4,6 \%)$, y los servicios comunitarios sociales y personales $(3,1 \%){ }^{6}$

6 Mensaje de fin de año del Presidente del Banco Central de Venezuela Nelson J. Merentes D. (2009).

Informe Gepec, Toledo, v. 18, n. 2, p. 137-146, jul./dez. 2014 
En este sentido el primer paso fue identificar las ramas (o sub-ramas) que se consideran productivas partiendo de la desagregación del PIB por sectores de origen en el Sistema de Cuentas Nacionales (SCN). Los sectores productivos con que se trabajó son: Actividades Agrícolas, Pecuarias y Caza; Industria Manufacturera; Construcción; Transporte, Almacenamiento y Comunicaciones; Explotación de Hidrocarburos, Minas y Canteras; Electricidad, Gas y Agua; Comercio, Restaurantes y Hoteles; se trata en primer lugar, de identificar las ramas que son primordialmente productivas y las que son primordialmente improductivas.

Por definición, usualmente se considera como improductivas las actividades de Comercio, Finanzas, Gobierno Central y una parte de Servicios Personales. Las ramas restantes se definen como productivas. ${ }^{7}$ Es importante aclarar que al interior de cada sector o rama productiva se despliegan trabajos y actividades que a su vez pueden ser calificadas como improductivas: publicidad, finanzas, vigilancia.

Se hace una mayor desegregación de cada rama. El punto es: en servicios hay actividades productivas (ej. salud) y otras improductivas (ej. publicidad, mercadeo, propaganda, etc.) y, por lo mismo, no se debe clasificar por completo a un sector (productivo) o al otro (improductivo).

A partir de la distinción por ramas y por funciones se necesita conocer la cantidad de personas ocupadas en la rama correspondiente para lo cual se toma como base la información obtenida en la Encuesta de Hogares por Muestreo Situación en la Fuerza de Trabajo Venezuela 2009 del Instituto Nacional de Estadística.

\subsection{Trabajo vivo social y la complejidad del trabajo}

Desde el punto de vista de las categorías más agregadas y desde la perspectiva de economía política, se analizó lo que Marx denominó "producto de valor" o lo que se conoce como "valor agregado" y en términos de valor, este indicador representa el trabajo vivo social que es reconocido como socialmente necesario. ${ }^{8}$

A los fines de manejar una aproximación al trabajo vivo, se calculó la diferencia entre población activa y el conjunto de trabajadores que desempeñan actividades que están al margen de la producción, es decir aquellos que se encuentran en la esfera improductiva. Este total equivale al total de productores directos. Esta última cifra se multiplicó por la jornada de trabajo con la cual se arriba a una expresión numérica que indicará la masa total de trabajo social productivo. Lo anterior se puede formalizar de la siguiente manera:

$(\mathrm{Tvd})=(\mathrm{N}) \cdot(\mathrm{Na} / \mathrm{N}) \cdot\left(\mathrm{Nap} / \mathrm{Na} \cdot(\mathrm{JT})^{9}\right.$

Donde:

Tvd: masa (en horas de trabajo vivo productivo disponible)

$\mathrm{N}$ : población total

7 Se hace referencia a la Teoría General del Trabajo Productivo, sin embargo, es preciso mencionar que no existe unanimidad en la conceptualización precisa del fenómeno que permita separar de manera tajante las actividades productivas e improductivas. Por tanto se hace referencia a lo dicho sobre actividades "primordialmente" productivas e improductivas para no hablar de "completamente" (Valenzuela, J. I:19). 8 Trabajo vivo social: entendido como la masa de trabajo disponible para fines de producción. 9 Valenzuela, J. (I:17).

Informe Gepec, Toledo, v. 18, n. 2, p. 137-146, jul./dez. 2014 
Na: población activa u ocupada

Nap: población activa productiva

JT: jornada de trabajo

Por otra parte, se necesita estimar la duración de la jornada de trabajo anual (en horas). Se supone, para este análisis, una jornada de trabajo anual de 2000 horas, tomando como referencia el supuesto utilizado usualmente (es el resultado de multiplicar la jornada de trabajo semanal por las semanas trabajadas al año). En la contabilización inicial y usual, sólo se considera la magnitud extensiva del trabajo gastado. O sea, su duración medida en horas astronómicas (horas reloj). Pero de inmediato surge la pregunta: ¿es correcto considerar la hora de trabajo en una actividad ultra compleja y difícil igual a la hora de trabajo que se gasta en lavar platos? Obviamente, no es lo mismo. Y se trata de homogeneizar la contabilización. Para lo cual, se debe considerar que el trabajo más complejo equivale a un múltiplo del más simple. Y viceversa. Por tanto, se considera la complejidad media y, en consecuencia, las horas de trabajo se "inflan" o "desinflan" según su complejidad sea superior o inferior a la media.

El problema que aquí surge es la inexistencia de información directa sobre la complejidad de los trabajos que se despliegan en el proceso económico. Para superar el inconveniente, se utiliza la calificación de la fuerza de trabajo y se hace un supuesto (algo heroico): que la complejidad se refleja bien en la calificación de la fuerza de trabajo. Luego, como segundo supuesto, se dice que la calificación depende de los años de educación formal. En la realidad, también influye los años de experiencia en el trabajo. Pero no hay tal información. Entonces, ¿a qué se llega?, a que en ciertas actividades un trabajador puede trabajar 100 horas pero sólo se reconocen 80 horas. Esto, en virtud de que el trabajo de dicho trabajador es más o menos complejo que el nivel medio. Además, como la complejidad y la calificación están relacionadas con el nivel de desarrollo, esto también indica que una economía de mercado premia el adelanto y castiga al atraso.

Para estimar los años de calificación media, para el conjunto del sector productivo se utiliza una media aritmética ponderada. $\mathrm{O}$ sea, ponderada por el peso de la rama en la ocupación total del sector productivo (más precisamente, en el total de horas de trabajo vivo productivo). Esto es, identificar los años de educación formal que, en promedio, posee la fuerza de trabajo que opera en la rama correspondiente.

Los elementos considerados para la calificación ramal del trabajo productivo son: nivel de instrucción, por años de estudio, población ocupada con instrucción y años de estudio, con estas variables se calcula la instrucción media de cada gran división económica. Así, la calificación media del sector productivo se obtuvo dividiendo la calificación media de cada rama entre el peso porcentual de la población económicamente activa (PEA).

El problema que aquí se presenta es la conversión del trabajo privado en trabajo social, para lo cual se considera para cada sector o rama el factor de conversión (fc) que resulta del utilizar las calificaciones medias ramales y dividirlas entre la calificación media del sector productivo. Este factor demuestra el nivel de desarrollo que tiene cada rama productiva en términos de trabajo social o lo que es lo mismo, el equilibrio que alcanza cada rama. Mientras mayor sea la agregación manejada, es más alta la probabilidad de que el factor de conversión se acerque más y más a uno y muy diferente de la unidad, cuando hay problemas de déficit de oferta o de sobreproducción. Para el 
presente análisis, se manejan cifras para un período relativamente "normal" y corto donde no tienen lugar ni grandes sobrantes ni grandes déficit de producción (Columna 3 , Cuadro 01).

Se conocen los años de educación formal en cada una de las ramas, para ejemplificar esta aproximación se denomina E1, E2 y E3. Se estiman los años de educación formal (promedio) para todo el sector productivo y se designa E.

Por lo tanto:

$\mathrm{E}=\mathrm{E} 1(\mathrm{~T} 1 / \mathrm{T})+\mathrm{E} 2(\mathrm{~T} 2 / \mathrm{T})+\mathrm{E}_{3}\left(\mathrm{~T}_{3} / \mathrm{T}\right)=\left[(\mathrm{E} 1)(\mathrm{T} 1)+\left(\mathrm{E}_{2}\right)(\mathrm{T} 2)+\left(\mathrm{E}_{3}\right)\left(\mathrm{T}_{3}\right)\right] / \mathrm{T}$

Luego, calcula el factor de conversión para cada rama:

$\mathrm{fc} 1=\mathrm{E} 1 / \mathrm{E} ; \mathrm{fc} 2=\mathrm{E} 2 / \mathrm{E} ; \mathrm{fc} 3=\mathrm{E} 3 / \mathrm{E}$

Conociendo tales factores se pasa a "homogeneizar" la hora de trabajo gastado. Para lo cual:

$\mathrm{TS} 1=\mathrm{T} 1(\mathrm{fc} 1) \quad \mathrm{TS} 2=\mathrm{T} 2(\mathrm{fc} 2) \quad \mathrm{TS} 3=\mathrm{T} 3(\mathrm{fc} 3)$

Y debe darse que: $\quad \mathrm{TS} 1+\mathrm{TS} 2+\mathrm{TS} 3=\mathrm{T} 1+\mathrm{T} 2+\mathrm{T}_{3}$

Teniendo todo lo anterior, se pasa a examinar el impacto del sector improductivo. Y calcular cuánto ingreso le come a los sectores productivos.

Para esto se puede tener que:

$\mathrm{T} 1=\mathrm{x} ; \quad \mathrm{T} 2=\mathrm{y} ; \quad \mathrm{T} 3=\mathrm{z} \quad \mathrm{T}=\mathrm{x}+\mathrm{y}+\mathrm{z}$

$\mathrm{E} 1=\mathrm{x} 1 ; \quad \mathrm{E} 2=\mathrm{x} 2 ; \quad \mathrm{E} 3=\mathrm{x} 3$

$\mathrm{E}=[\mathrm{x} \cdot \mathrm{x} 1+\mathrm{x} \cdot \mathrm{x} 2+\mathrm{x} \cdot \mathrm{x} 3] / \mathrm{T}$

$\mathrm{fc} 1=\mathrm{x} 1 / \mathrm{E} ; \mathrm{fc} 2=\mathrm{x} 2 / \mathrm{E} ; \mathrm{fc} 3=\mathrm{x} 3 / \mathrm{E}$

$\mathrm{TS} 1=\mathrm{fc} 1 . \mathrm{T} 1 ; \mathrm{TS} 2=\mathrm{fc} 2 . \mathrm{T} 2 ; \mathrm{TS} 3=\mathrm{fc} 3 \cdot \mathrm{T} 3$

$\mathrm{TS}=\mathrm{TS} 1+\mathrm{TS} 2+\mathrm{TS} 3=\mathrm{E}$

Informe Gepec, Toledo, v. 18, n. 2, p. 137-146, jul./dez. 2014 
Cuadro 01 - Trabajo Vivo Social

\begin{tabular}{|c|c|c|c|c|}
\hline Rama & Ocupación (1) & \begin{tabular}{|c|} 
Factor de \\
Conversión \\
$(2)$ \\
\end{tabular} & $\begin{array}{c}\text { Ocupación } \\
\text { "Social“" } \\
(\mathbf{3})=(1)(2) \\
\end{array}$ & $\begin{array}{c}\text { Trabajo Vivo } \\
\text { Social (miles } \\
\text { horas) (3)(JT)* } \\
\end{array}$ \\
\hline Actividades Agrícolas, pecuarias y caza & 1.025 .035 & 0,250 & 256.259 & 512.517 .500 \\
\hline Industria Manufactura & 1.406 .774 & 0,980 & 1.378 .639 & 2.757.277.040 \\
\hline Construcción & 1.088 .895 & 0,650 & 707.782 & 1.415 .563 .500 \\
\hline $\begin{array}{l}\text { Transporte, almacenamiento y } \\
\text { comunicaciones }\end{array}$ & 1.029 .022 & 0,800 & 823.218 & 1.646 .435 .200 \\
\hline $\begin{array}{l}\text { Explotación de hidrocarburos, minas y } \\
\text { canteras }\end{array}$ & 107.422 & 0,920 & 98.828 & 197.656 .480 \\
\hline Electricidad, gas y agua & 58.730 & 1,050 & 61.667 & $123 \cdot 333.000$ \\
\hline Comercio, restaurantes y hoteles & 2.811 .622 & 0,900 & 2.530 .460 & 5.060 .919 .600 \\
\hline Total & $7 \cdot 527.500$ & 5,550 & 5.856 .851 & 11.713 .702 .320 \\
\hline
\end{tabular}

* Se supone una Jornada de Trabajo anual de 2000 horas.

Fuente: Elaboración Propia a partir de datos aportados por el INE, Sistema de Cuentas Nacionales y Valenzuela J. (2007). Cuentas Nacionales y Categorías de la Economía Política. (2011)

\subsection{Cálculo de las transferencias de valor}

Hasta este momento se manejó el criterio de Smith, el más estrecho. Y en los tiempos actuales, esa aproximación ya no es la más oportuna. En este sentido, entender desde la visión marxista el proceso de las transferencias de valor, supone considerar lo que es el valor agregado generado o producido y el valor agregado apropiado. Para ello, se puede decir en términos muy simples que el valor producido, es aquel que ha elaborado la rama o sector y el valor apropiado es el que queda en poder de esa rama o sector.

Para obtener el valor agregado producido por cada rama se necesita conocer el trabajo vivo total, el cual se relaciona con el Producto Interno Neto a costo de factores (o en su defecto con el PIB a precios de mercado). En este caso se utiliza el PIB a precios de mercado para obtener el cociente que define la "expresión monetaria del valor" (emw), el cual señala la cantidad de unidades monetarias que por hora de trabajo social está manejando el sistema, es decir, el reciproco del valor del dinero (Columna 3, Cuadro 02). La "expresión monetaria del valor" se obtiene dividiendo el valor agregado bruto monetario (según Cuentas Nacionales) por el trabajo vivo social total. Se expresa: emw= $\mathrm{VAB} / \mathrm{Tvs}$. 
Cuadro 02 - Valor Producido y Apropiado

\begin{tabular}{|c|c|c|c|c|c|c|}
\hline Rama & $\begin{array}{c}\text { Trabajo Vivo } \\
\text { Social (1) }\end{array}$ & $\begin{array}{c}\text { Expresión } \\
\text { Monetaria } \\
\text { del Valor } \\
\text { (2)* }\end{array}$ & \begin{tabular}{|c|} 
Valor \\
Agregado \\
Producido \\
(millones) \\
$(3)=(1)(2)$ \\
\end{tabular} & $\begin{array}{l}\text { Valor Agregado } \\
\text { Apropiado } \\
\text { (millones) (4) }\end{array}$ & $\begin{array}{c}\text { Coeficiente } \\
\text { F (4/3) }\end{array}$ & 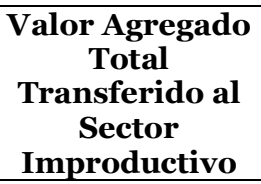 \\
\hline $\begin{array}{l}\text { Actividades } \\
\text { Agrícolas, pecuarias } \\
\text { y caza }\end{array}$ & 512.517 .500 & 0,056 & 28.589 .570 & 2.628 .001 & 0,092 & 25.961 .569 \\
\hline $\begin{array}{l}\text { Industria } \\
\text { Manufactura }\end{array}$ & 2.757 .277 .040 & 0,056 & 153.808 .144 & $9 \cdot 347.534$ & 0,061 & 144.460 .610 \\
\hline Construcción & 1.415 .563 .500 & 0,056 & 78.963 .844 & 4.318 .676 & 0,055 & 74.645 .168 \\
\hline $\begin{array}{l}\text { Transporte, } \\
\text { almacenamiento y } \\
\text { comunicaciones }\end{array}$ & 1.646 .435 .200 & 0,056 & 91.842 .473 & 5.282 .328 & 0,058 & 86.560 .145 \\
\hline $\begin{array}{l}\text { Explotación de } \\
\text { hidrocarburos, minas } \\
\text { y canteras }\end{array}$ & 197.656 .480 & 0,056 & 11.025 .797 & 5.890 .838 & 0,534 & 5.134 .959 \\
\hline $\begin{array}{l}\text { Electricidad, gas y } \\
\text { agua }\end{array}$ & 123.333 .000 & 0,056 & 6.879 .838 & 1.336 .757 & 0,194 & $5 \cdot 543.081$ \\
\hline $\begin{array}{l}\text { Comercio, } \\
\text { restaurantes y } \\
\text { hoteles }\end{array}$ & 5.060 .919 .600 & 0,056 & 282.311 .366 & 935.732 .398 & 3,315 & -653.421 .032 \\
\hline Total & 11.713 .702 .320 & - & 653.421 .032 & 964.536 .532 & 4,308 & -311.115 .500 \\
\hline
\end{tabular}

*Precios Corrientes.

Fuente: Elaboración Propia a partir de datos aportados por el INE, Sistema de Cuentas Nacionales y Valenzuela J. (2007). Cuentas Nacionales y Categorías de la Economía Política. (2011)

Ahora bien, una parte del nuevo valor que genera la economía es el transferido desde el sector productivo al improductivo. La porción que se transfiere se ve representada como el coeficiente "g" (Columna 2, Cuadro 03) y se obtiene de la diferencia entre el valor agregado total y el total del valor producido por cada rama. Para la economía venezolana (considerando los sectores escogidos para el análisis) el coeficiente "g" sería igual a 0.476 , es decir, un $47.6 \%$ del producto agregado total es apropiado por el sector improductivo.

El impacto de los sectores improductivos, medidos por "g", siempre será negativo. A partir de esto, se mide también el coeficiente global, definido como " $\mathrm{f}$ " (Columna 3, Cuadro 03) para representar el nivel del valor apropiado con respecto al producido. En este sentido se espera que como regla, el coeficiente " $\mathrm{f}$ " sea menor que uno (1), es decir, que el valor apropiado sea inferior al producido. (Cuadro o3).

Cuadro 03 - Transferencias de Valor

\begin{tabular}{l|cc}
\hline \multicolumn{1}{c|}{ Rama } & $\mathbf{g}$ & $\mathbf{f}$ \\
\hline Actividades Agrícolas, pecuarias y caza & $-0,476$ & 0,092 \\
Industria Manufactura & $-0,476$ & 0,061 \\
Construcción & $-0,476$ & 0,055 \\
Transporte, almacenamiento y comunicaciones & $-0,476$ & 0,058 \\
Explotación de hidrocarburos, minas y canteras & $-0,476$ & 0,534 \\
Electricidad, gas y agua & $-0,476$ & 0,194 \\
Comercio, restaurantes y hoteles & $-0,476$ & 3,315 \\
\hline Total & $-0,476$ & 4,308 \\
\hline
\end{tabular}

Fuente: Elaboración Propia a partir de datos aportados por el INE, Sistema de Cuentas Nacionales y Valenzuela J. (2007). Cuentas Nacionales y Categorías de la Economía Política (2011).

Informe Gepec, Toledo, v. 18, n. 2, p. 137-146, jul./dez. 2014 


\section{Consideraciones Finales}

En este artículo se presenta una clasificación de algunos sectores primordialmente improductivos en la economía venezolana, a saber: Comercio, Restaurantes y Hoteles; Electricidad, Agua y Gas; y parte del sector de Comunicaciones referido a servicios personales. Mientras que los sectores de Actividades Agrícolas, Pecuarias y Caza; Manufactura; Construcción; Explotación de Hidrocarburos, Minas y Canteras se definen como productivas. En el caso venezolano solo el sector de Comercio, Restaurantes y Hoteles escapa del drenaje global de excedentes y opera (por lo menos para el año 2009) con un valor apropiado superior al producido. Los sectores más perjudicados por las transferencias de valor son Manufactura, Construcción e Hidrocarburos, con un coeficiente menor que "1". En Manufactura y Construcción, el alto nivel de drenaje de valor que experimentan, se ve asociado a la influencia del Estado en las políticas de estímulo hacia estos sectores. La condición rentística de la economía influencia de manera directa el comportamiento en el sector de Hidrocarburos. Es importante destacar que según los aproximaciones derivadas de los cálculos, el drenaje fuerte es el que se genera hacia los sectores improductivos, vale destacar un 47,6\% del producto agregado generado es desviado hacia esos sectores. Los resultados obtenidos reflejan un nivel de productividad sujeto a la generación de valor agregado tomado de algunos sectores, es decir, el indicador de Valor Agregado Nacional, solo muestra un porcentaje sumado de todos los sectores, sin desagregar el origen de ese valor. El proceso de planificación bajo este contexto, está sujeto a la medición y consideración de los valores transferidos, para trabajar en base a la realidad productiva de cada sector. Con esta primera aproximación se evidencia que la promoción del valor agregado nacional se encuentra supeditada a la apropiación que el sector improductivo realiza del valor generado por los sectores productivos. Se considera demostrada la viabilidad de la Teoría del Valor-trabajo para fundamentar las transferencias de valor de las actividades productivas a las improductivas.

\section{Referencias}

BANCO CENTRAL DE VENEZUELA. Mensaje de fin de año del Presidente del Banco Central de Venezuela. Nelson J. Merentes D. Caracas. 2009.

BAPTISTA, Asdrúbal. Itinerario por la Economía Política. Caracas: Ediciones IESA: Academia Nacional de Ciencias Económicas, 2008.

DOS SANTOS, Theotonio. Imperialismo y Dependencia, ERA, México, 1978.

INSTITUTO NACIONAL DE ESTADÍSTICA. Encuesta de Hogares por Muestreo, Situación en la Fuerza de Trabajo Venezuela 2009. Caracas, 2009.

MARX, Karl. El Capital. Crítica de la Economía Política. Tercera Edición, tercera Reimpresión. Fondo de Cultura Económica. México, 2006.

PROYECTO NACIONAL SIMÓN BOLÍVAR 2007-2013. 
RUBÍN, I. Ensayos sobre la Teoría Marxista del Valor; Pasado y Presente, Buenos Aires, 1974.

VAlenZUEla, José. Producto, Excedente y Crecimiento. El Sistema de Fuerzas Productivas. Departamento de Economía. Universidad Autónoma Metropolitana. Iztapalapa. México, 2009.

VALENZUELA, José. Cuentas Nacionales y Categorías de la Economía Política. Departamento de Economía. Universidad Autónoma Metropolitana. Iztapalapa. México, (s/f).

Submetido em 19/05/2014.

Sobre a autora

\section{Luciana Madrid Cobeña}

Economista, docente-investigadora da Escuela Venezolana de Planificación, Ministerio del Poder Popular para la Planificación y Finanzas, Caracas, (0058) 0426-1053804.

Email:1mcm6o@hotmail.com 